\title{
Back-scattered electron imaging of a non-vertebral case of hypervitaminosis $A$ in a cat
}

\author{
J Franch ${ }^{1}$, J Pastor ${ }^{2}$, B Franch ${ }^{1}$, I Durall ${ }^{1}$, M C Manzanares $^{3}$
}

${ }^{1}$ Surgery and ${ }^{2}$ Medicine, Veterinary School of Barcelona, 08193 Bellaterra

${ }^{3}$ Unitat d'Anatomia, Pavello Central, Planta 5 Campus de Bellvitge, Universitat de Barcelona, 08907 Hospitalet, Barcelona, Spain

Date accepted: 7 February 2000

\begin{abstract}
We describe a clinical case of hypervitaminosis A in a cat. The main lesions were bony fusions of both the hip and stifle joints, without spinal involvement. A post-mortem study using back-scattered scanning electron microscopy (BEI-SEM) revealed that exostoses had formed around the joints without articular surface involvement. The more recently formed areas of bony proliferation were composed mainly of chondroid tissue surrounded by different degrees of woven bone. As the bony reaction occurred, remodelling of the trabeculae was observed which lead to progressive substitution of chondroid tissue by woven bone surrounded by apposition of lamellar bone. No traces of calcified cartilage were observed in any of the bone sections evaluated.
\end{abstract}

$\mathrm{H}$ ypervitaminosis $\mathrm{A}$ is a metabolic bone disease associated with a chronic excess of vitamin A which is frequently described as a result of feeding a diet consisting largely of liver. The condition, first described by English \& Seawright (1964), mainly affects cats but a few experimentally induced (Cho et al 1975) or clinical (Stewart 1965) cases have been described in the dog. The excessive intake of vitamin A results in dystrophic calcification of the axial and appendicular skeleton. The radiographic changes can be detected as early as 14 weeks after beginning the diet (Seawright et al 1967), although the usual onset of the disease is insidious, from months to years after the diet was first started (Bennett 1976).

Classical clinical signs of hypervitaminosis A include neck stiffness, with painful lesions in the early stages of the disease, followed by vertebral ankyloses causing abnormal posture. Other associated clinical signs are lethargy, depression, irritability, poor grooming, lameness and gingivitis (Johnson et al 1995). The treatment necessitates avoiding the source of vitamin A to prevent the development of further exostoses; however, it may be difficult to persuade affected cats to eat anything other than liver (Lecoteur \& Child 1995).

Correspondence: Jordi Franch DVM PhD
Exostoses of the cervicothoracic vertebrae are the most frequent radiographic finding observed in cats with hypervitaminosis A, although the limb joints are also affected in some cases (Armstrong \& Hand 1989). Osteophyte formation and ankyloses of the spine and limb joints have not been well studied in the cat. It has been hypothesised that this bone formation may reflect increased periosteal sensitivity to the effect of tension at sites of tendon, ligament and joint capsule attachments (Seawright et al 1967). The type of bone formation in these areas has not to our knowledge been studied previously in the cat. The objectives of this paper are (1) to describe a clinical case of hypervitaminosis A with involvement of the hindlimbs but no spinal involvement and (2) to study the different types of calcified tissues involved around the ankylosed joints.

\section{Materials and methods}

An 8-year-old female domestic shorthair cat was presented to the Veterinary Teaching Hospital of the Autonomous University of Barcelona with hindlimb paresis. From an early age, the cat had consumed a diet composed of raw liver and commercial cat food. The physical examination revealed bilateral ankylosis of the hip and stifle joints and a poorly groomed coat. 


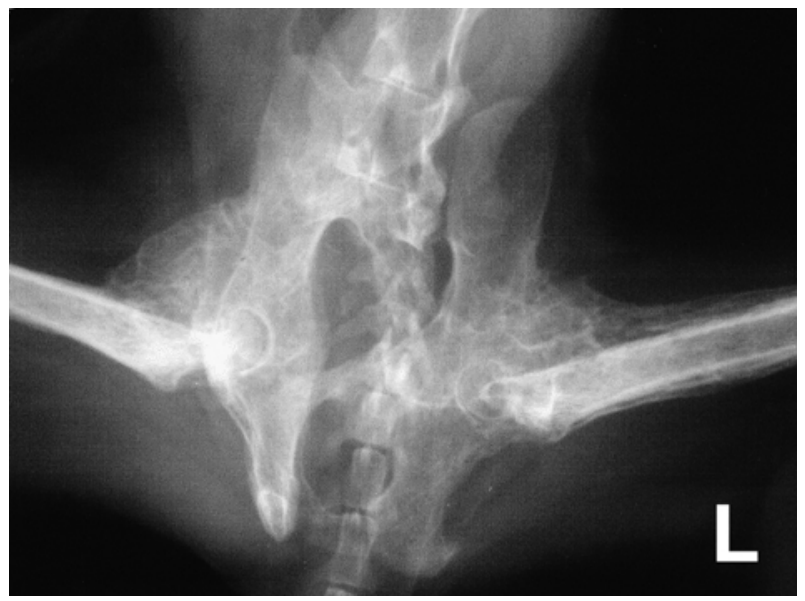

Fig 1. Slight oblique ventrodorsal pelvic radiograph showing bilateral bone proliferation on the craniodorsal aspect of both hip joints.

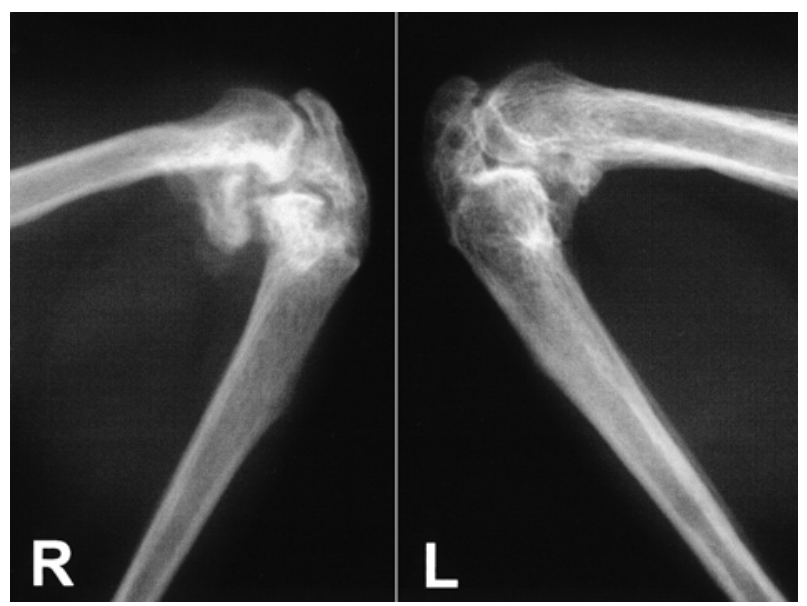

Fig 2. Mediolateral radiograph of both stifle joints showing the massive bone exostoses.

The most severe skeletal lesions were observed radiographically in both hindlimbs. In the ventrodorsal pelvic radiograph, a massive bony proliferation was observed around both hip joints which was more developed in the dorsocranial aspects of the joints (Fig 1). In the lateral radiographs, a highly dense well-developed bony mass was seen cranially and dorsally to the acetabulum. Ankylosis prevented extension of either stifle and only lateral views were obtained. These demonstrated a complete mineralisation of the patellar ligaments and bony exostoses also bridged both stifle joints caudally (Fig 2). The confluent exostoses around both hip and stifle joints were so severe and extensive that the original joint surfaces were difficult to identify and a true bony arthrodesis of these joints had developed.
No other radiographic findings were observed in the axial or appendicular skeleton of the cat despite some mild exostoses on the ventral aspect of the second, third and fourth sternebrae and on the third and fourth left costal cartilages and costochondral junctions.

Hypervitaminosis A was diagnosed on the basis of the dietary history and clinical and radiographic findings. Due to the advanced stage of the disease and the poor condition of the cat the owners elected to have the animal euthanased.

For the back-scattered scanning microscopy study, longitudinal $2 \mathrm{~mm}$ width sections were obtained from the right hip and stifle macerated joints, where lesions appeared macroscopically to be most severe. The hip joint sections were oriented lateromedially and the stifle joint sections were oriented cranio-caudally. The sections were not decalcified and were fixed, dehydrated, embedded in polymethylmethacrylate and ground according to the method described by Donath (1988). The specimens were then coated with evaporated carbon and studied using a scanning electron microscope provided with a backscattered-electron detector. Backscattered-electron imaging using a scanning electron microscope (BEI-SEM) is considered to be a useful technique for determining the relative differences between calcified tissues from bone samples (Skedros et al 1993).

\section{Results}

\section{Gross anatomical findings}

At postmortem examination, dissection and maceration of the affected bones confirmed the complete bony arthrodesis observed radiographically. A compact bony mass covering both the hip and the stifle joints was the most obvious macroscopic finding. Although both hip joints were severely affected, the bony mass was larger on the right side and occupied the whole area between the ventral aspect of the body of the ilium, the greater trochanter and the cranial aspect of the proximal quarter of the femoral shaft (Fig 3).

Both stifle joints were completely bridged by new bone formation. Although the left side showed a more compact and advanced degree of joint fusion, the right bony proliferation was larger, especially on the medial aspect, and presented a more irregular surface (Fig 4). The 


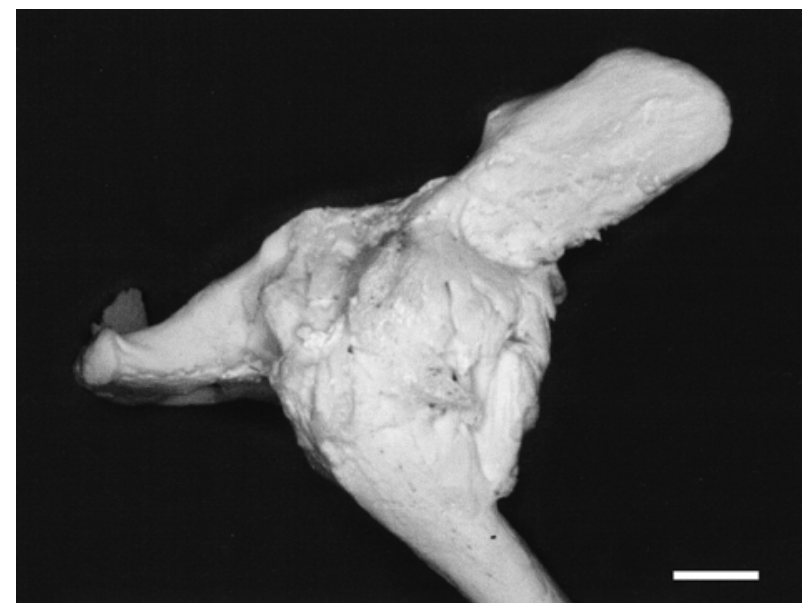

Fig 3. Lateral aspect of the macerated right pelvis and femur showing the extensive bony reaction involving the hip joint (bar $=1 \mathrm{~cm}$ ).

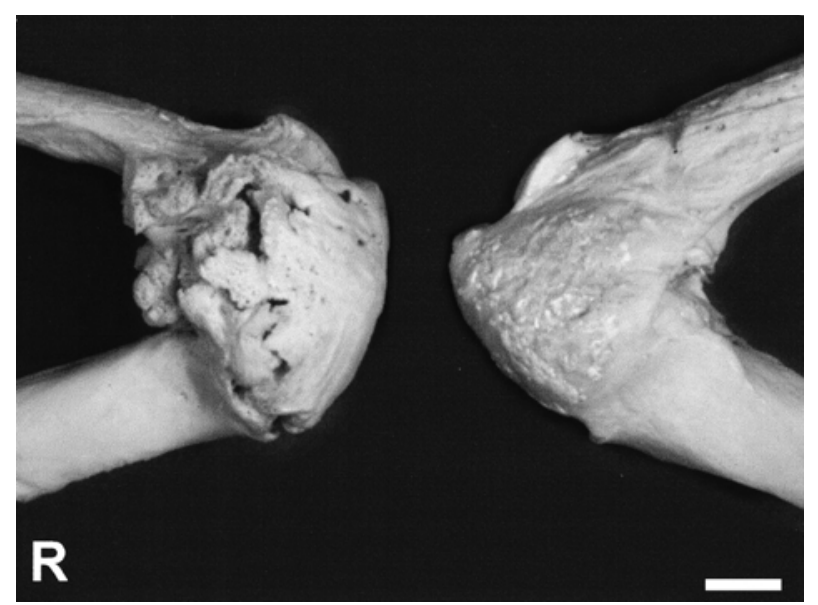

Fig 4. Medial aspects of both macerated right (R) and left stifle joints showing the severe bony proliferation $(\mathrm{bar}=1 \mathrm{~cm})$.

newly formed bone covered both joints from the patellar apex to the tibial tuberosity, including the patellar ligament. The base of the patella remained unaffected and no signs of calcification were observed in the tendinous insertion of the quadriceps femoralis muscle (Fig 4). Neither the hip nor stifle joints showed any mobility.

Examination of the macerated thorax demonstrated a mild bony proliferation between the more cranial sternebrae and a more severe exostoses in the costochondral junction of the left third and fourth ribs. No evidence of bony proliferation was observed in the spine or in other limb joints.

\section{BEI-SEM findings}

Hip joint. The BEI-SEM study of the hip joint sections demonstrated that the complete bone

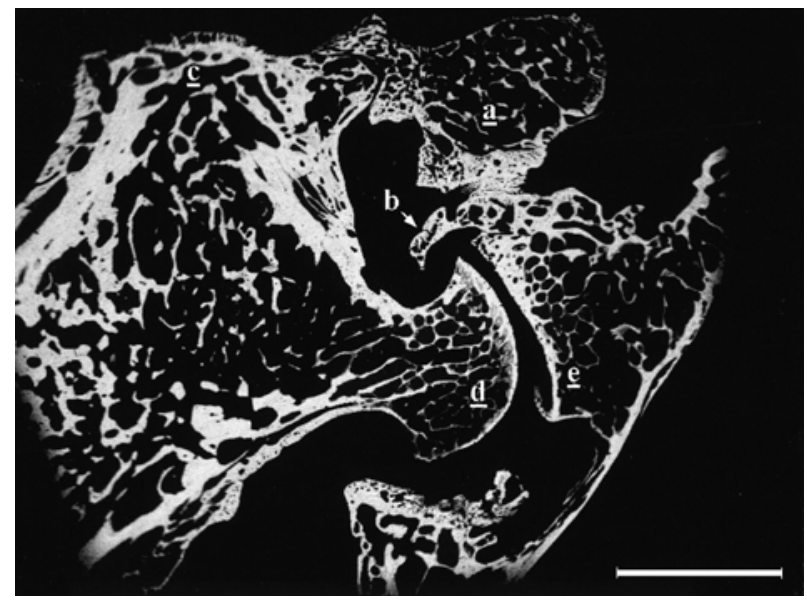

Fig 5. BEI-SEM aspect of the hip joint area. External (a) and internal (b) bony bridges were developed from the supraacetabular area to the proximal femur. The ventral bone proliferation was located distally to the area shown in this picture. Greater trochanter (c), femoral head (d) and acetabulum (e) (bar=5 mm).

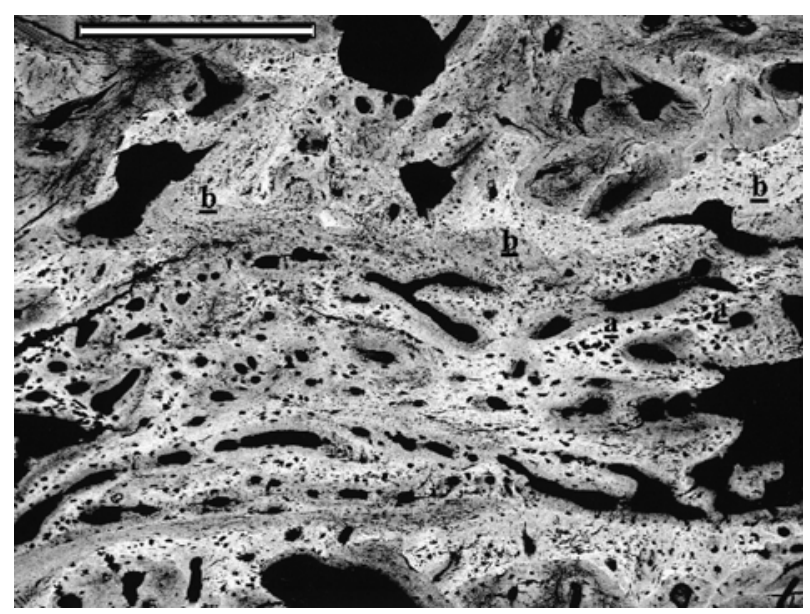

Fig 6. BEI-SEM aspect of the bony trabeculae from the external bony bridge near the acetabulum. Note the chondroid tissue cores (white areas) surrounded by newly formed woven bone (grey areas). Chondroid tissue (a); woven bone (b) (bar $=500 \mu \mathrm{m})$.

bridging of the joint was extra-articular, extending from the greater trochanter to the cranial and dorsal acetabular rim without fusion between the articular surfaces (Fig 5). A second dorsal bone bridge, parallel to the external one, was apparently forming closer to the articular surface. A ventral osseous bridge, situated a few millimeters distally from the ventral acetabular rim, was evident in the more cranial bone sections.

The whole inner bridge as well as the areas closer to the greater trochanter and acetabulum of the outer bridge were formed from a compact, 


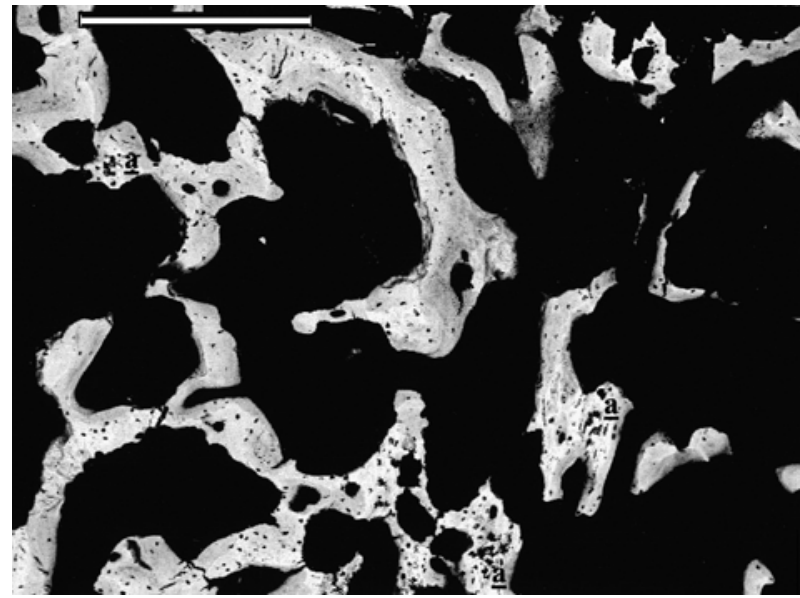

Fig 7. BEI-SEM aspect of the trabeculae from the dorsal area of the external bony bridge far from the acetabulum and greater trochanter. The sparse bone trabeculae are mainly formed by woven bone with a few central cores of chondroid tissue (a) (bar $=500 \mu \mathrm{m})$.

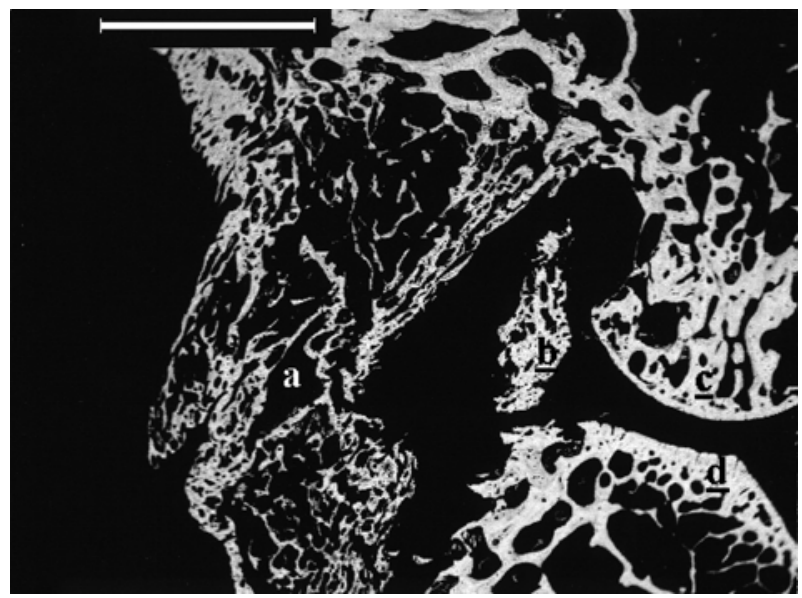

Fig 8. BEI-SEM micrograph of the caudal aspect of the right stifle showing the outer (a) and inner (b) osseous proliferation bridging the stifle joint. Lateral femoral condyle (c) and tibial plateau $(\mathrm{d})(\mathrm{bar}=5 \mathrm{~mm})$.

well-mineralised trabecular network of chondroid tissue and woven bone. The whitest (most highly calcified) areas of chondroid tissue were completely surrounded by a dense grey mass of woven bone, with an apparently random arrangement of the trabeculae (Fig 6). The trabecular density clearly decreased in the more dorsal area of the external bridge callus, further from the greater trochanter and acetabulum (Fig 7). These trabeculae were also made up of central areas of chondroid tissue surrounded by apposition of woven bone with some traces of lamellar bone. Nevertheless, the ratio of woven bone to chondroid tissue was clearly greater in this area compared to that in the more compact areas

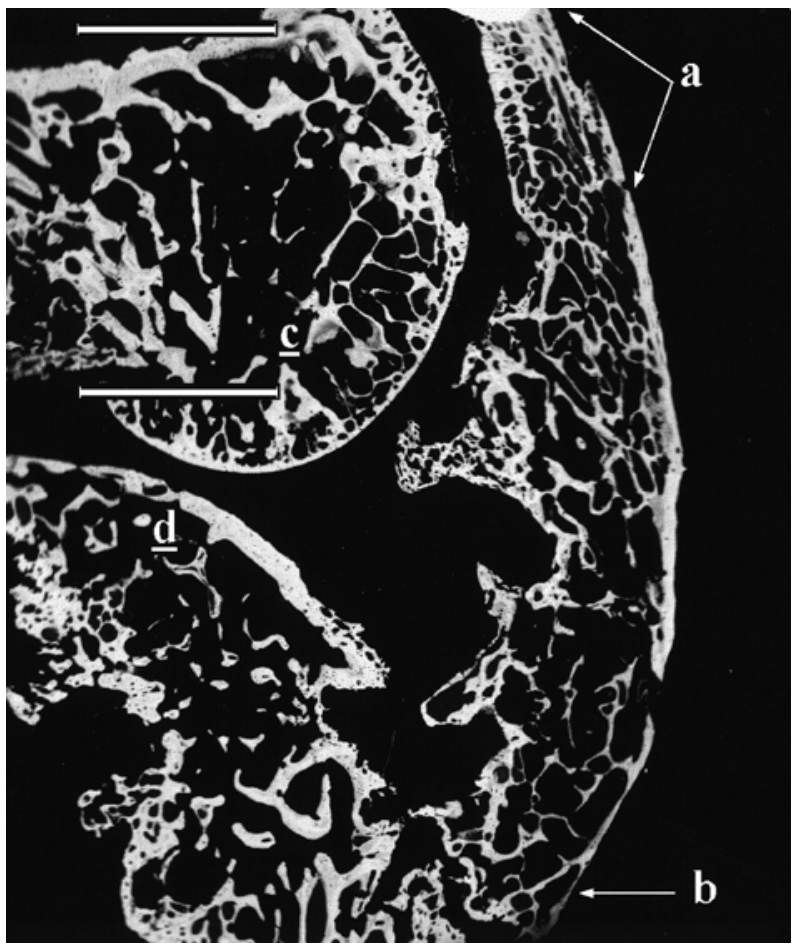

Fig 9. BEI-SEM micrographs showing the cranial aspect of the right stifle. Note the complete calcification of the tibiopatellar ligament from the patella (a) to the tibial tuberosity (b). Medial femoral condyle (c) and tibial plateau (d) (bar $=5 \mathrm{~mm}$ ).

of the external bone bridge near the trochanter and acetabulum (Fig 6).

Stifle joint. The BEI-SEM study of the stifle joint sections demonstrated a complete extra-articular bony bridging of the joint. In the caudal aspect of the joint, the bony proliferation extended from the distal femoral shaft to the proximal tibia (Fig 8). An external bony bridge, located about $5 \mathrm{~mm}$ caudally to the joint, and a second inner bony bridge, just caudal to the joint surface, were fused in the more medial bony sections. In the cranial aspect of the joint, a complete ossification of the patellar ligament from the patellar apex to the tibial tuberosity was evident (Fig 9). The proliferation of bone was expanding from the calcified patellar ligament into the triangular intra-articular area cranial to both the femoral and tibial articular surfaces (Fig 9). In the more lateral bone sections, this triangular area was almost completely occupied by the bony ingrowth, although the articular surfaces of both the femur and tibia, and even the patella, were apparently affected slightly or not at all. The femoral and tibial bone cortices in contact with the bony proliferation appeared to be severely 


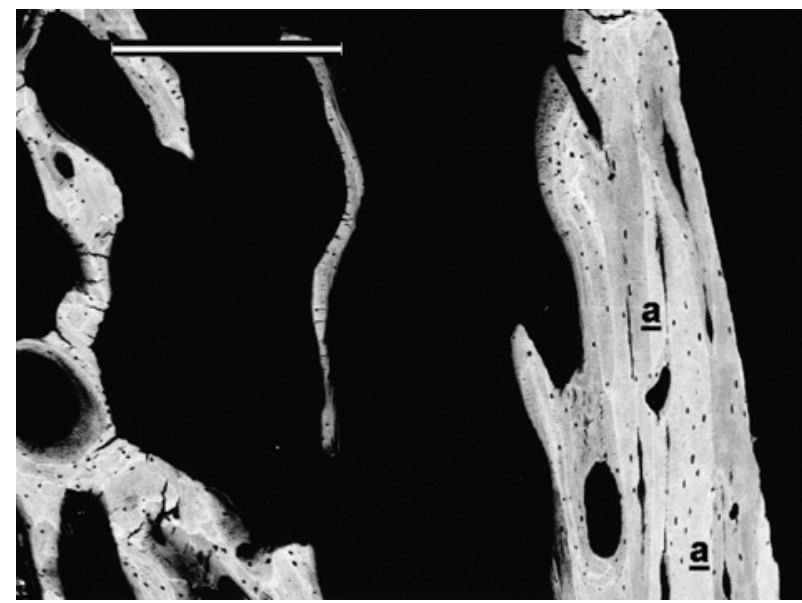

Fig 10. BEI-SEM aspect of the calcified tibiopatellar ligament next to the patellar insertion. Note the well-oriented disposition of the lamellar bone trabeculae, especially at the cranial aspect of the ligament. Lamellar bone (a) (bar= $500 \mu \mathrm{m})$.

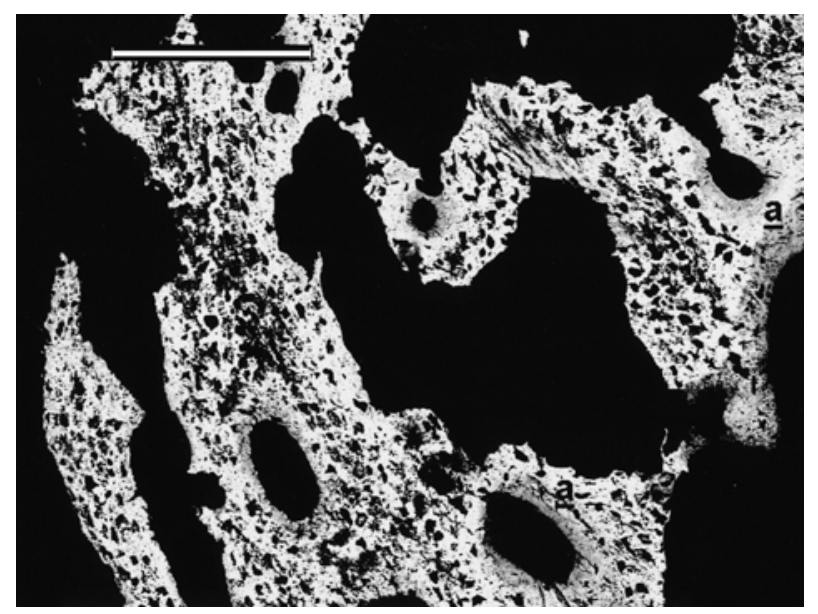

Fig 11. The BEI-SEM aspect of the middle level of the calcified ligament and the intra-articular bony mass was characterised by the presence of bone trabeculae formed mainly by chondroid tissue with very little woven bone apposition (a) (bar $=200 \mu \mathrm{m})$.

altered, losing the normal architectural pattern of cortical bone (Fig 8). Similarly, the distal half of the patella showed increased bone porosity, whereas the proximal half showed a quite normal compact bone pattern.

At higher magnification, clear differences in trabecular composition were evident between the different areas of bony proliferation. In the proximal calcified patellar ligament the trabeculae were composed mainly of lamellar bone with some traces of woven bone (Fig 10). The trabeculae of the lamellar bone, especially in the cranial aspect, were clearly oriented following the long axis of the patellar ligament. At the middle level

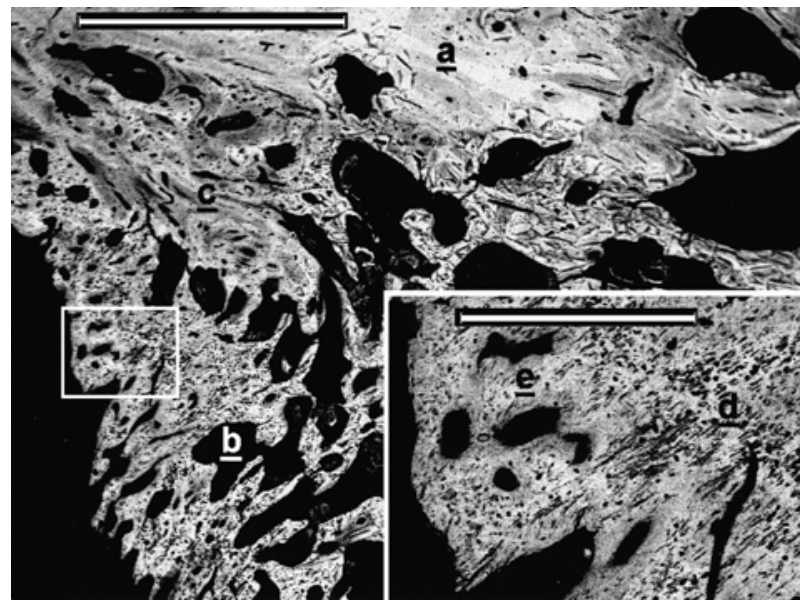

Fig 12. BEI-SEM aspect of the transitional area between the original femoral caudal cortex (a) and the caudal osseous bridge (b). The more proximal area of the bone proliferation, completely integrated on the femoral cortex, was mainly formed by woven bone (c). This bone pattern is progressively changing distally to a pattern formed by central cores of chondroid tissue (d) surrounded by a moderate amount of woven bone (e) as observed at higher magnification (inset) (bar=2 mm; inset bar $=500 \mu \mathrm{m}$ ).

of the calcified ligament, as well as in the whole bony mass growing up into the joint space, the main calcified tissue was chondroid tissue with very little apposition of woven bone (Fig 11). The rest of the calcified patellar ligament was formed mainly from chondroid tissue cores surrounded by a different degree of woven bone apposition. A different pattern was observed only in the more cranial aspect of the calcified patellar ligament, where a thin layer of lamellar bone was present, and at the insertion of the ligament on the tibial tuberosity, where the calcified tissue was mainly woven bone with a few central areas of chondroid tissue.

The transitional area between the caudal bony bridge and the original femoral cortex was characterised by the above-mentioned loss of the cortical bone pattern and the presence of large quantities of woven bone with some lamellar bone apposition without a well-defined orientation (Fig 12). More distally, the dense bony mass was composed mainly of chondroid tissue surrounded by a moderate amount of woven bone (Fig 12). The high density of bony proliferation changed into a more sparse trabecular arrangement at the middle level of the caudal bony bridge. In this area, the outer trabeculae were formed mainly from chondroid tissue surrounded by some traces of woven bone, while the inner trabeculae were made up almost completely from woven bone. 
Despite the severe bony lesions, the articular space was preserved in all the stifle bone sections in a similar fashion to the hip joint sections.

\section{Discussion}

Hypervitaminosis A was originally named 'feline deforming cervical spondylosis' (English \& Seawright 1964) because extensive confluent exostoses of the cervicothoracic vertebrae are the most frequent radiographic finding. In addition to the vertebral lesions, bony exostoses involving the shoulders, elbows and other limb joints may also be present (Armstrong \& Hand 1989). Softtissue calcification has also been reported in some affected cats and it can be associated with a less intense, more chronic hypervitaminosis $\mathrm{A}$ in the mature animal (Bennett 1976). Very few cases have been described without vertebral involvement (Vanderlip 1983). The case described in this paper did not present vertebral lesions and despite the mild sternal bony proliferation, the most important lesions were severe, bilateral bony exostoses bridging both the hip and the stifle joints with complete patellar ligament calcification.

The effects of vitamin A toxicosis on bone seem to differ between different species. A histological study on calcified bone samples was performed on growing rats following experimentally induced hypervitaminosis A (Hough et al 1988). Their most important findings were bone weakness and spontaneous fractures due to an increased bone resorption, osteoclastosis and a paucity of trabecular surfaces covered with osteoid. The authors considered that the bone lesions could be explained, at least in part, by a direct effect of vitamin A on skeletal tissue. This was based on the fact that vitamin A selectively inhibited in vitro collagen synthesis in a dose-dependent fashion (Hough et al 1988).

Excessive intake of vitamin $\mathrm{A}$ is involved in the aetiopathogenesis of 'hyena disease' in cattle (Carroll Woodward et al 1997). The process is characterised by closure of the growth plate, especially in the distal femur and proximal tibia, due to suppression of chondrocyte and osteoblast differentiation and proliferation (Takaki et al 1996). Similar findings have recently been described in experimentally induced hypervitaminosis $\mathrm{A}$ in growing rats (Kodaka et al 1998). Although growth plate closure was not observed by Tang et al (1985) after oral administration of toxic levels of vitamin A for 21 consecutive days in broiler and leghorn chickens, the authors reported other types of growth plate disturbances as well as parathyroid gland hyperplasia, thinning of the osteogenic layer of the periosteum and generalised osteoporosis.

Osteoporosis is the main bone lesion observed in humans after long-term administration of vitamin A for oncological or dermatological diseases (Hough et al 1988, DiGiovanna et al 1996). A recent study has demonstrated that only some retinoids (ie, etretinate) are directly involved in the pathogenesis of human hypervitaminosis $\mathrm{A}$ (DiGiovanna et al 1996). Only one case has been described with acetabular and spine hyperostosis after 6 years of vitamin A treatment in a patient with hyperlipidaemia although the authors did not consider vitamin A toxicosis to be the main cause of the bony lesions (Romero et al 1996).

Despite the growth disturbances observed in young cats with experimentally induced hypervitaminosis A (Clark \& Seawright 1968), the bony lesions in other species have very little or no similarity to the common lesions observed in clinical cases of feline hypervitaminosis A. Osteoporosis and bone weakness is described occasionally (Bennett 1976) but it is considered to have an origin in lack of use. On the other hand, it appears unlikely that toxic levels of vitamin A inhibit collagen synthesis in cats, as has been proposed for rats (Hough et al 1988), as the feline proliferative lesions would require active production of collagen for new bone formation. Furthermore, the thin periosteal osteogenic layer observed in the histological study of Tang et al (1985) appears unlikely to be involved in the pathogenesis of feline hypervitaminosis A, considering the periosteal origin of the bony exostoses observed in our BEI-SEM study.

The BEI-SEM identification of the different calcified tissues observed in our study was based on the back-scattered electron contrast and fibre arrangement of the matrix as well as the size and shape of the cellular lacunae, as previously described by Franch et al (1998a). Very little information is available regarding histological bone changes in feline hypervitaminosis A for comparison of our BEI-SEM results with those from other microscopic studies. Some authors have reported a subperiosteal origin of bone proliferation (Seawright et al 1967) with apposition of new woven bone around the apophyseal joints of the cervical and thoracic vertebrae and proliferation of cartilage from the joint margins (Pedersen et al 1983). Woven bone was also 
present in our BEI-SEM observations, although it was not the only bony tissue observed. The other calcified tissues observed in our study were chondroid tissue and lamellar bone, although no traces of calcifying cartilage were detected either in the hip or stifle bone sections. In one recent report, calcified cartilage was found during the closure of growth plates in a BEI-SEM study of experimentally induced hypervitaminosis A in growing rats (Kodaka et al 1998).

The calcified pattern of the trabeculae which formed the bony proliferation appears to be standardised. The areas which were apparently more recently formed were composed mainly of chondroid tissue with a small degree of woven bone. As the bony mass grows, a remodelling process occurs which leads to a progressive substitution of chondroid tissue by woven bone, surrounded by lamellar bone apposition. The amount of lamellar bone present will depend on the stage of the remodelling process, so that the older areas of the bony exostoses could be identified depending on the amounts of woven and lamellar bone present. This trabecular remodelling pattern is very similar to that observed in the fracture healing process (Franch et al 1998a) or in other bone diseases such as craniomandibular osteopathy (Franch et al 1998b). It is probable that any periosteal reaction follows an identical and well-defined ossification process independent of the aetiology of the condition. Based on this assumption, the bony exostoses first formed in both stifle joints and the hip joints were affected later. Due to the high apposition of lamellar bone, it can be suggested that the first stifle area involved in the bony proliferation was close to the patellar origin of the patellar ligament. This is also the sole area affected radiologically in cats with hypervitaminosis A with mild stifle joint involvement (Armstrong \& Hand 1989). It would appear that the bony proliferation occurs in some soft tissues, eg, the patellar ligament in the cranial aspect of the stifle joint and joint capsule and probably the gluteal tendons at the hip joint. The inner part of the bony bridges described in our study, which affected both hip and the stifle joints caudally, apparently followed the course of the corresponding joint capsules. Although the reason for limb-joint involvement in cats with hypervitaminosis A remains unknown, we consider that several factors must be involved in addition to the increase of the sensitivity of periosteum to the effects of trauma proposed by some authors (Seawright et al 1967).
Further investigation of the species-related factors will be important in explaining the differences between the macroscopic and microscopic bony lesions in clinical feline hypervitaminosis A and vitamin A toxicosis in other species and to elucidate why cats appear to be especially susceptible to this disease.

\section{Acknowledgements}

We wish to thank the BSE-SEM team at the 'Serveis Cientifico-Tècnics' of the Universitat de Barcelona for their technical and scientific advice.

\section{References}

Armstrong PJ, Hand MS (1994) Nutritional disorders. In: The Cat. Diseases and Clinical Management (2nd edn), Scherding RG (ed.). Churchill Livingstone, New York, pp. 1639-1640

Bennett D (1976) Nutrition and bone diseases in the dog and cat. The Veterinary Record 98, 313-320

Carroll Woodard J, Donovan AG, Eckhoff C (1997) Vitamin (A and D)-induced premature closure (hyena disease) in calves. Journal of Comparative Pathology 116, 353-366

Clark L, Seawright AA (1968) Skeletal abnormalities in the hindlimbs of young cats as a result of hypervitaminosis A. Nature 217, 1174-1176

Cho DY, Frey RA, Guffy MM (1975) Hypervitaminosis A in the dog. American Journal of Veterinary Research 36, 1597-1599

DiGiovanna JJ, Sollitto RB, Abangan DL, Steinberg SM, Reynolds JC (1996) Osteoporosis is a toxic effect of longterm entretinate therapy. Archives of Dermatology 131, 1263-1267

Donath K (1988) Preparation of Histologic Sections by the Cutting-Grinding Technique for Hard Tissue and Other Material not Suitable to be Sectioned by Routine Methods. Equipment and Methodical Performance. Norderstedt, Germany: Exakt-Kulzer-Publication

English PB, Seawright AA (1964) Deforming cervical spondylosis of the cat. Australian Veterinary Journal 40, 376-379

Franch J, García F, Camón J, Manzanares MC (1998a) Backscattered electron imaging of the calcified tissues present in bone healing. Veterinary and Comparative Orthopaedics and Traumatology 2, 105-111

Franch J, Cesari JR, Font J (1998b) Craniomandibular osteopathy in two Pyrenees mountain dogs. The Veterinary Record 142, 455-459

Hough S, Avioli LV, Muir H, Gelderblom D, Jenkins G, Kurasi H, Slatopolsky E, Bergfeld MA, Teitelbaum SL (1998) Effects of hypervitaminosis A on the bone and mineral metabolism of the rat. Endocrinology 122, 2933-2939

Johnson KA, Watson ADJ, Page RL (1995) Skeletal diseases. In: Textbook of Small Animal Internal Medicine, Ettinger SJ, Feldman EC (eds). W.B. Saunders, Philadelphia, pp. 20772103

Kodaka T, Takaki H, Soeta S, Mori R, Naito Y (1998) Local disappearance of epiphyseal growth plates in rats with hypervitaminosis A. Journal of Veterinary Medicine Science 60, 815-821 
Lecouteur RA, Child G (1995) Diseases of the spinal cord. In: Textbook of Veterinary Internal Medicine (4th edn), Ettinger SD, Feldman EC (eds). WB Saunders, Philadelphia, pp. 629-695

Pedersen NC, Pool RR, Morgan JP (1983) Joint diseases of dogs and cats. In: Textbook of Veterinary Internal Medicine (2nd edn), Ettinger SJ (ed.). WB Saunders, Philadelphia, pp. 2187-2235

Romero JB, Schreiber A, Von Hochstetter AR, Wagenhauser FJ, Michel BA, Theiler R (1996) Hyperostotic and destructive osteoarthritis in a patient with vitamin A intoxication syndrome: a case report. Bulletin-Hospital for Joint Diseases 54, 169-174

Seawright AA, English PB, Gartner RJW (1967) Hypervitaminosis $\mathrm{A}$ and deforming cervical spondylosis of the cat. Journal of Comparative Pathology 77, 29-39
Skedros JG, Bloebaum RD, Bachus KN, Boyce TM (1993) The meaning of gray levels in back-scattered electron images of bone. Journal of Biomedical Material Research 27, 47-50

Stewart RJC (1965) Canine and Feline Nutritional Requirements, Graham-Jones O. (ed.). Pergamon Press, Oxford

Takaki H, Fukuda S, Mori R, Kodaka T, Sato R, Naito Y (1996) Changes in bone metabolism and epiphysial growth plate in bovine Hyena disease induced by administration of vitamin AD3E premix or Vitamin A. Journal of Veterinary Medical Science 58, 407-412

Tang KN, Rowland GN, Veltmann JR Jr (1985) Vitamin A toxicity: comparative changes in bone of the broiler and leghorn chicks. Avian Diseases 29, 416-429

Vanderlip SL (1983) What is your diagnosis? Journal of American Veterinary Medical Association 183, 1472-1474 